\title{
Interleukin-17A and B-cell activating factor in chronic hepatitis C patients with or without asymptomatic mixed cryoglobulinemia: effects of antiviral treatment and correlations with vitamin D
}

\author{
Polydoros Konstantinides, Alexandra Alexopoulou, Emilia Hadziyannis, Theoni Kanellopoulou, \\ Spyridon P. Dourakis \\ Medical School, National and Kapodistrian University of Athens, Hippokration General Hospital, Athens, Greece
}

\section{Abstract}

\begin{abstract}
Background Several studies have provided conflicting results regarding the immune responses in chronic hepatitis $\mathrm{C}(\mathrm{CHC})$ patients with mixed cryoglobulinemia (MC). The importance of B-cell activating factor (BAFF) in MC has been described, but the role of interleukin (IL)-17A is less clear.

Methods Serum concentrations of IL-17A, BAFF and 25-OH vitamin D were measured in CHC patients at baseline, end of treatment, and 6 months post-treatment with pegylated interferon- $\alpha$ and ribavirin, versus 12 healthy controls.

Results Thirty-four patients (20 male, mean age $40.7 \pm 9.2$ years, 12 of genotype 1 or 4,22 of genotype 2 or 3 ) were included, of whom $64.7 \%$ achieved a sustained virological response (SVR). MC was detected in $52.9 \%$ of the patients. Higher levels of both cytokines were found in patients with MC compared to those without. Patients who achieved SVR had higher pretreatment IL-17A and lower BAFF levels compared to those without SVR. IL-17A was downregulated during and following treatment in responders, whereas upregulation was observed in non-responders. $\mathrm{CHC}$ patients demonstrated low vitamin D levels compared to HC. Moreover, the changes in IL-17A over the treatment period were significantly associated with vitamin $\mathrm{D}$ changes $(\beta=-0.04, \mathrm{SE}=0.02$, $\mathrm{P}=0.046)$. No difference in IL-17A, BAFF and vitamin $\mathrm{D}$ values was seen between patients with cirrhosis $(n=14)$ and those without.
\end{abstract}

Conclusions $\mathrm{CHC}$ patients with asymptomatic MC have increased levels of IL-17A and BAFF. IL$17 \mathrm{~A}$ levels decline significantly while BAFF increases during treatment in responders. An interplay between IL-17A and vitamin D concentrations was revealed during the antiviral treatment.

Keywords Interleukin 17A, B-cell activating factor, vitamin D, chronic hepatitis C, mixed cryoglobulinemia

Ann Gastroenterol 2018; 31 (6): 1-7

\section{Introduction}

Globally, the prevalence of chronic hepatitis $\mathrm{C}(\mathrm{CHC})$ patients is about $2.5 \%$, corresponding to $>177$ million infections

2nd Department of Internal Medicine and Research Laboratory, Medical School, National and Kapodistrian University of Athens, Hippokration General Hospital, Athens, Greece

Conflict of interest: None

Correspondence to: Polydoros Konstantinides, 15 Eynardou St, 15237 Athens, Greece, e-mail: polidorosk@hotmail.com

Received 8 June 2018; accepted 23 August 2018; published online 14 September 2018

DOI: https://doi.org/10.20524/aog.2018.0310 worldwide [1]. Mixed cryoglobulinemia (MC) has been recognized as the most common extrahepatic manifestation of hepatitis $\mathrm{C}$ virus (HCV) infection. It is an autoimmune disorder characterized by the presence of circulating cold-precipitating immune complexes [2]. These complexes are detected with a prevalence of $40-50 \%$ in CHC patients, but most of them are asymptomatic [3].

The intrinsic mechanisms inducing cryoglobulin production are still unclear. A common hypothesis is that chronic stimulation by $\mathrm{HCV}$ antigens, mainly the HCV core antigen, facilitates the expansion of rheumatoid factorproducing B lymphocytes [4]. It is possible that both cytokines and the host's genetic background may also contribute to lymphoproliferation in $\mathrm{CHC}[5,6]$. B-cell activating factor (BAFF), also called B-lymphocyte stimulator (BLyS), has been identified as a novel ligand of the tumor-necrosis factor 
superfamily. It is produced by dendritic cells, monocytes, macrophages and activated $\mathrm{T}$ lymphocytes, and stimulates survival of B lymphocyte and autoantibody production [7]. Antiviral treatment with interferon has been shown to significantly increase BAFF levels [8].

Previous studies have also demonstrated that T-cell immunoregulatory cytokines contribute to liver damage [9] and that HCV may directly modulate both B- and T-cell functions [10]. Among immune deregulations, those related to subtype 17 of the T-helper lymphocyte (Th17)/interleukin-17 (IL-17) axis have been recognized as key immune-pathological and prognostic elements in patients with chronic viral hepatitis. The proinflammatory cytokine IL-17A may activate neutrophils, leading to tissue inflammation and fibrosis, and promote the progression of autoimmune ddiseases $[11,12]$. Recently-developed direct acting antiviral agents (DAAs) have achieved excellent therapeutic results in $\mathrm{CHC}$ patients. However, DAA therapy may not be adequate for the treatment of $\mathrm{MC}$ and combination with immunosuppressive therapy may be necessary $[13,14]$. Notably, limited data are available on the efficacy of antiviral treatment in HCV-related cryoglobulinemia [15]. On the other hand, due to financial reasons, pegylated interferon- $\alpha$ (pegIFNa) and ribavirin (RBV) therapy is still the standard treatment for $\mathrm{CHC}$ patients in some countries [16].

Vitamin D is a critical regulator of immunity, playing a role in both innate and cell-mediated immune responses [17]. Numerous studies have demonstrated a decline in vitamin D concentrations in CHC patients [18], but the causes of this deficiency have not yet been defined. Moreover, vitamin D deficiency is considered as a risk factor of fibrosis and treatment failure [19].

The objectives of this study were to investigate the kinetics of IL-17A and BAFF in HCV patients with or without MC, at baseline (BSL) and during the antiviral treatment, and to evaluate their interactions with viral clearance, vitamin D levels, and fibrosis.

\section{Patients and methods}

Consecutive $\mathrm{CHC}$ patients were prospectively evaluated from March 2011 to March 2014 in the Hepatology Department of Hippokration General Hospital, Athens, Greece. The study protocol was approved by the Medical School of Athens and the local Ethics Committee of Hippokration General Hospital.

$\mathrm{CHC}$ was confirmed by HCV RNA polymerase chain reaction (PCR) and $\mathrm{HCV}$ genotyping was performed in all subjects. Inclusion criteria were: a) written informed consent provided by the patients; b) age $\geq 18$ years (without any upper age limit); and c) chronic HCV infection. Exclusion criteria were: a) coinfection with hepatitis B virus or human immunodeficiency virus; b) symptomatic cryoglobulinemia with vasculitis; and c) decompensated cirrhosis.

Forty-six individuals who met the inclusion and exclusion criteria were initially evaluated, of whom 34 were ultimately judged eligible for enrollment. Twelve healthy individuals matched for age and sex were used as the control group (HC). All patients received pegIFNa/RBV treatment for 48 weeks (genotype 1 and 4 ) or 24 weeks (genotype 2 and 3 ).

The following parameters were recorded from each patient participating in the study:

i) HCV viral load, quantified by real-time HCV PCR assay with a lower limit of quantification of $13 \mathrm{IU} / \mathrm{mL}$ (Versant kPCR, Siemens, Tarrytown, NY, USA) and HCV genotype (line probe assay, VERSANT HCV Genotype 2.0, Siemens, Tarrytown, NY, USA).

ii) Cryoglobulins: Blood samples of approximately $20 \mathrm{~mL}$ were collected into pre-warmed tubes and clotted at $37^{\circ} \mathrm{C}$ for $>1 \mathrm{~h}$. The serum was separated from clots by centrifugation at $3000 \mathrm{rpm}$ for $20 \mathrm{~min}$ and was incubated for 7 days at $4^{\circ} \mathrm{C}$. The presence of cryoglobulin was first assessed by visual inspection of a precipitate and was confirmed by its dissolution at $37^{\circ} \mathrm{C}$. The cryoglobulin concentration was measured after centrifugation as the cryocrit.

iii) Liver stiffness was assessed by transient elastography (TE) and patients with a TE $>12.5 \mathrm{kPa}$ were identified as cirrhotic.

iv) Complete blood count and biochemistry panel, including 25-OH vitamin $\mathrm{D}$, using immunoassay kit.

v) Abdominal ultrasound: Groups were categorized by: 1) the presence or not of $\mathrm{MC}$ at BSL; 2) response to treatment (with or without sustained virological response, SVR); 3) the presence or not of cirrhosis; and 4) level of viremia at BSL with low or high HCV-RNA level (after interpretation of our data we used a cut-off point of 400,000 IU/ $\mathrm{mL}$, similarly to other studies, showing an improved discrimination between high and low probability of achieving an SVR) [20].

Blood samples were obtained at three time points: before starting treatment (BSL), at the end of treatment (EOT), and 6 months after the end of treatment (6Mo-EOT). Blood was collected in tubes with ethylenediamine tetraacetic acid and centrifuged at $800 \mathrm{~g}$ at room temperature. Plasma was collected, aliquoted and stored at $-80^{\circ} \mathrm{C}$ until assayed. Concentrations of IL-17A and BAFF were measured using commercial enzymelinked immunosorbent assay (ELISA) kits according to the manufacturer's instructions (eBioscience, human BAFF, instant ELISA, BMS2007INST and eBioscience, human IL-17A platinum ELISA, BMS2017TEN).

\section{Statistical analysis}

All continuous variables are presented as mean \pm standard deviation. Quantitative variables are presented with absolute and relative frequencies. Spearman's correlation coefficients were used to explore the association of two continuous variables. Chi-square test was used for the comparison of proportions. Differences between different groups in changes of study variables during the follow-up period were evaluated using repeated measurements analysis of variance (ANOVA). Bonferroni correction was used in case of multiple testing. IL-17A and BAFF had skewed distribution and were log-transformed for the analysis. To longitudinally assess if changes in BAFF, IL-17A and vitamin D 
during follow up were correlated, mixed linear regression models were fitted, accounting for multiple measurements per individual obtained at different time points. Regression coefficients $(\beta)$ with standard errors (SE) were computed from the results of the mixed models. All P-values reported are two-tailed. Statistical significance was set at 0.05 and analyses were conducted using SPSS statistical software (version 22.0).

\section{Results}

Twenty male and 14 female patients, mean age $40.7 \pm 9.2$ years were included. Patients' characteristics are presented in Table 1. Twelve cases had genotype 1 or 4 and 22 genotype 2 or

Table 1 Sample characteristics

\begin{tabular}{lc}
\hline Characteristic & $\mathrm{N}(\%)$ \\
\hline Sex & $20(58.8)$ \\
Male & $14(41.2)$ \\
Female & $40.7 \pm 9.2$ \\
Age, mean \pm SD & \\
Nationality & $24(70.6)$ \\
Greek & $10(29.4)$ \\
Other & $25.8 \pm 4.4$ \\
BMI, mean \pm SD & $55 \pm 28.6 / 85 \pm 54$ \\
AST/ALT, mean \pm SD & \\
HCV-RNA IU/mL (baseline) & $11(32.4)$ \\
$<400,000$ & $23(67.6)$ \\
$>400,000$ & \\
Genotype & $12(35.3)$ \\
1+4 & $22(64.7)$ \\
$2+3$ & \\
MC & $16(47.1)$ \\
No & $18(52.9)$ \\
Yes & \\
\hline MC, mixed cryoglobulinemia; SD, standard deviation; BMI, body mass \\
index; AST, aspartate aminotransferase; ALT, alanine aminotransferase; \\
HCV-RNA, hepatitis C virus ribonucleic acid
\end{tabular}

3. Cryoglobulins were detected in $52.9 \%$ of the cases. Twenty two patients $(64.7 \%)$ achieved SVR and the rate was higher in HCV genotypes 2 and 3 (77.3\%) compared to genotypes 1 and $4(41.7 \%)(\mathrm{P}=0.003)$. Additionally, the percentage of patients who achieved SVR was similar in those with and without MC (62.5\% vs. $66.7 \%, \mathrm{P}=0.8)$. Cirrhosis $(\mathrm{TE}>12.5 \mathrm{kPa})$ was found in 14 patients.

The mean BSL IL-17A values in patients with $\mathrm{CHC}$ were higher than in $\mathrm{HC}(\mathrm{P}=0.020)$. The mean $\mathrm{BAFF}$ values tended to be higher in $\mathrm{CHC}$ patients compared to $\mathrm{HC}(\mathrm{P}=0.076)$. Low vitamin $\mathrm{D}$ concentrations were measured in $\mathrm{CHC}$ patients (mean: $22.21 \pm 8.87 \mathrm{ng} / \mathrm{mL})$. Vitamin D deficiency $(<20 \mathrm{ng} / \mathrm{mL})$ or insufficiency (20-29 ng/mL) at BSL was observed in $55 \%$ and $17 \%$ of patients, respectively.

The changes in IL-17A, BAFF and vitamin D over time are illustrated in Table 2. IL-17A remained unchanged, while BAFF increased significantly at the EOT and vitamin D decreased significantly at $6 \mathrm{Mo}-\mathrm{EOT}$. However, no significant change in vitamin $\mathrm{D}$ was found between BSL and 6Mo-EOT values.

Table 3 shows the changes in IL-17A, BAFF and vitamin D levels over the study period in relation to SVR. At BSL, patients with SVR had significantly higher IL-17A $(\mathrm{P}=0.040)$ and lower BAFF levels $(\mathrm{P}=0.045)$ compared to those with no SVR. IL-17A declined significantly from BSL to EOT in the SVR group and this decline persisted thereafter. In contrast, IL-17A increased significantly from BSL to EOT in subjects without SVR ( $\mathrm{P}=0.022)$. The IL-17A kinetics during the follow-up period differed significantly $(\mathrm{P}=0.001)$ between those with and without SVR (Fig. 1). BAFF was significantly elevated in responders compared to non-responders; this elevation persisted after treatment discontinuation only in responders but it did not reach statistical significance. The kinetics of BAFF levels differed significantly $(\mathrm{P}=0.01)$ over the study period between responders and non-responders (Fig. 2). Vitamin D declined significantly only from EOT to $6 \mathrm{Mo}$-EOT in patients with SVR.

Values and changes in IL-17A, BAFF and vitamin D levels in relation to the presence of $\mathrm{MC}$ are presented in Table 4. Patients with MC had significantly higher IL-17A and BAFF levels compared to those without. No significant differences in the three parameters were found between the MC and non-MC groups over the total period of treatment and follow up.

Results from mixed linear models showed that changes in BAFF were not significantly associated with the corresponding

Table 2 Values of IL-17A (pg/mL), BAFF (ng/mL) and vitamin D (ng/mL) levels in all HCV patients and changes during the therapy

\begin{tabular}{|c|c|c|c|c|c|c|c|c|c|}
\hline \multirow[t]{2}{*}{ Parameter } & \multicolumn{5}{|c|}{ Mean \pm SD } & \multicolumn{4}{|c|}{ Significance } \\
\hline & BSL & EOT & 6Mo-EOT & $\begin{array}{c}\text { Change BSL } \\
\text { to EOT }\end{array}$ & $\begin{array}{l}\text { Change EOT } \\
\text { to } 6 \mathrm{Mo}-\mathrm{EOT}\end{array}$ & $\mathrm{P}^{1}$ & $\mathrm{P}^{2}$ & $\mathrm{P}^{3}$ & $\mathrm{P}^{4}$ \\
\hline IL-17A & $16.6(31.95)$ & $9.66(22.02)$ & $8.67(22.6)$ & $-6.94(18.35)$ & $-0.99(6.09)$ & 1.000 & 0.700 & 1.000 & 0.392 \\
\hline $\mathrm{BAFF}^{+}$ & $0.11(0.31)$ & $0.3(0.62)$ & $0.34(1.07)$ & $0.19(0.54)$ & $0.04(0.57)$ & 0.043 & 0.991 & 0.366 & 0.004 \\
\hline Vit. D & $22.21(8.87)$ & $22.93(7.21)$ & $18.01(6.91)$ & $0.72(8.39)$ & $-4.92(5.87)$ & 1.000 & 0.011 & 0.167 & 0.002 \\
\hline
\end{tabular}


Table 3 Values of IL-17A (pg/mL), BAFF (ng/mL) and vitamin D (ng/mL) levels in patients with and without SVR and changes during therapy

\begin{tabular}{|c|c|c|c|c|c|c|c|c|c|}
\hline \multirow[t]{2}{*}{ Parameter } & \multicolumn{5}{|c|}{ Mean \pm SD } & \multicolumn{4}{|c|}{ Significance } \\
\hline & BSL & EOT & 6MA-EOT & $\begin{array}{c}\text { Change BSL to } \\
\text { EOT }\end{array}$ & $\begin{array}{l}\text { Change EOT } \\
\text { to 6MA-EOT }\end{array}$ & $\mathrm{P}^{2}$ & $\mathrm{P}^{3}$ & $\mathrm{P}^{4}$ & $\mathrm{P}^{5}$ \\
\hline \multicolumn{10}{|l|}{$\mathrm{IL}^{-17^{+}}$} \\
\hline \multicolumn{10}{|l|}{ SVR } \\
\hline No & $4.58(12.37)$ & $7.42(6.92)$ & $7.17(9.06)$ & $2.84(12.05)$ & $-0.25(7.36)$ & 0.020 & 1.000 & 0.076 & 0.001 \\
\hline Yes & $23.15(37.37)$ & $10.88(27.06)$ & $9.48(27.53)$ & $-12.27(19.2)$ & $-1.4(5.42)$ & 0.022 & 0.591 & 0.016 & \\
\hline $\mathrm{P}^{1}$ & 0.040 & 0.799 & 0.489 & & & & & & \\
\hline \multicolumn{10}{|l|}{$\mathrm{BAFF}^{+}$} \\
\hline \multicolumn{10}{|l|}{ SVR } \\
\hline No & $0.24(0.5)$ & $0.28(0.5)$ & $0.19(0.43)$ & $0.04(0.21)$ & $-0.09(0.12)$ & 1.000 & 0.453 & 1.000 & 0.010 \\
\hline Yes & $0.03(0.07)$ & $0.32(0.68)$ & $0.42(1.3)$ & $0.28(0.64)$ & $0.1(0.69)$ & 0.017 & 1.000 & 0.089 & \\
\hline $\mathrm{P}^{1}$ & 0.045 & 0.978 & 0.652 & & & & & & \\
\hline \multicolumn{10}{|l|}{ Vitamin D } \\
\hline \multicolumn{10}{|l|}{ SVR } \\
\hline No & $19.55(6.57)$ & $20.84(4.02)$ & $18.36(4.02)$ & $1.28(6.14)$ & $-2.48(4.66)$ & 1.000 & 0.419 & 1.000 & 0.029 \\
\hline Yes & $23.66(9.74)$ & $24.06(8.33)$ & $17.83(8.16)$ & $0.41(9.52)$ & $-6.24(6.14)$ & 1.000 & $<0.001$ & 0.001 & \\
\hline $\mathrm{P}^{1}$ & 0.202 & 0.218 & 0.834 & & & & & & \\
\hline
\end{tabular}

${ }_{1}^{1}$-value for group effect; ${ }^{2} P$-value for time effect (BSL to end of therapy measurement); ${ }^{3} P$-value for time effect (end of therapy to 6 months measurement); ${ }^{4} P$-value for time effect (BSL to 6 months measurement); ${ }^{5}$ Repeated measurements ANOVA. Effects reported include significant differences between the two groups in the degree of change in each particular variable during the study period; ${ }^{+}$analysis was based on logarithmic transformations, BSL, baseline; EOT, end of therapy; 6Mo-EOT, 6 months after the end of therapy; IL-17A, interleukin 17A; BAFF, B-cell activating factor; SVR, sustained virological response

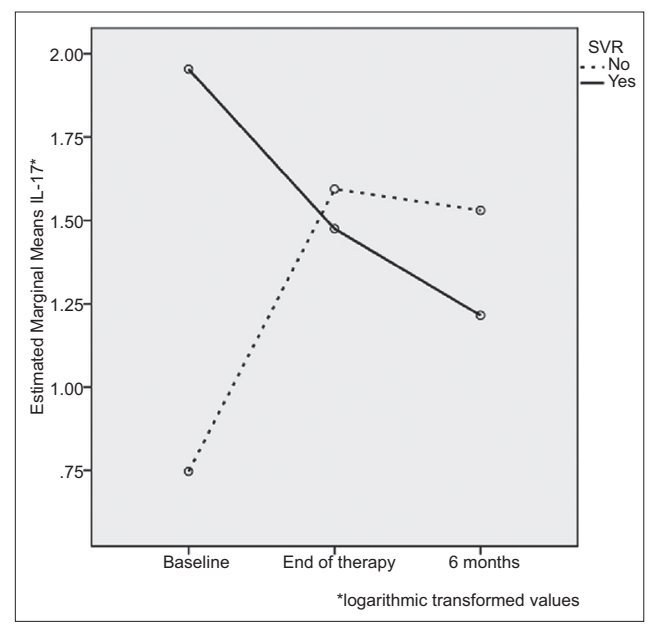

Figure 1 Changes in interleukin 17A levels during follow up in relation to sustained virological response (SVR)

changes in IL-17A $(\beta=-0.23, \mathrm{SE}=0.44, \mathrm{P}=0.601)$. Additionally, no significant association of vitamin $\mathrm{D}$ changes with corresponding BAFF changes was demonstrated during follow-up $(\beta=0.82, \mathrm{SE}=2.59, \mathrm{P}=0.751)$. In contrast, it was found that IL-17A changes were significantly associated with vitamin $D$ changes $(\beta=-0.04, S E=0.02, P=0.046)$.

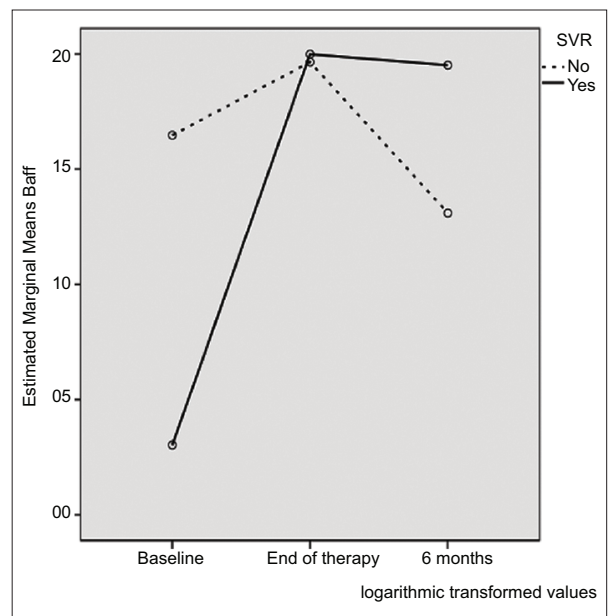

Figure 2 Changes in B-cell activating factor levels during follow up in relation to sustained virological response (SVR)

Correlation coefficients between IL-17A, BAFF and vitamin $\mathrm{D}$ showed that vitamin $\mathrm{D}$ levels at $\mathrm{BSL}$ were negatively correlated with BAFF at EOT $(\mathrm{P}=0.017)$ and at $6 \mathrm{MoA}-\mathrm{EOT}$ $(\mathrm{P}=0.03)$. No correlation was found between vitamin $\mathrm{D}$ and $\mathrm{BAFF}$ at BSL or between vitamin D and IL-17A at any time point. Finally, no significant differences in IL-17A, BAFF 
Table 4 Values of IL-17A (pg/mL), BAFF (ng/mL) and vitamin D (ng/mL) levels in patients with and without MC and changes during therapy

\begin{tabular}{|c|c|c|c|c|c|c|c|c|c|}
\hline \multirow[t]{2}{*}{ Parameter } & \multicolumn{5}{|c|}{ Mean \pm SD } & \multicolumn{4}{|c|}{ Significance } \\
\hline & BSL & EOT & 6Mo-EOT & $\begin{array}{c}\text { Change BSL to } \\
\text { EOT }\end{array}$ & $\begin{array}{c}\text { Change EOT to } \\
6 \mathrm{Mo} \text { EOT }\end{array}$ & $\mathrm{P}^{2}$ & $\mathrm{P}^{3}$ & $\mathrm{P}^{4}$ & $\mathrm{P}^{5}$ \\
\hline \multicolumn{10}{|l|}{ IL17 $^{+}$} \\
\hline \multicolumn{10}{|l|}{$\mathrm{MC}$} \\
\hline No & $6.73(10.85)$ & $4.2(4.42)$ & $4.17(8.02)$ & $-2.52(9.1)$ & $-0.03(6.42)$ & $1.000^{+}$ & $0.730^{+}$ & $1.000^{+}$ & $0.855^{+}$ \\
\hline Yes & $25.38(41.29)$ & $14.51(29.51)$ & $12.67(29.98)$ & $-10.86(23.36)$ & $-1.85(5.83)$ & $1.000^{+}$ & $1.000^{+}$ & $1.000^{+}$ & \\
\hline $\mathrm{P}^{1}$ & $0.021^{+}$ & $0.180^{+}$ & $0.079^{+}$ & & & & & & \\
\hline \multicolumn{10}{|l|}{$\mathrm{BAFF}^{+}$} \\
\hline \multicolumn{10}{|l|}{ MC } \\
\hline No & $0.02(0.04)$ & $0.11(0.23)$ & $0.06(0.15)$ & $0.09(0.23)$ & $-0.05(0.18)$ & 0.903 & 1.000 & 1.000 & 0.351 \\
\hline Yes & $0.18(0.42)$ & $0.47(0.79)$ & $0.58(1.44)$ & $0.29(0.7)$ & $0.1(0.76)$ & 0.047 & 1.000 & 0.243 & \\
\hline $\mathrm{P}^{1}$ & 0.047 & 0.071 & 0.093 & & & & & & \\
\hline \multicolumn{10}{|l|}{ vitD } \\
\hline \multicolumn{10}{|l|}{ MC } \\
\hline No & $21.34(10.44)$ & 22.07 (8.69) & $16.99(7.91)$ & $0.73(7.64)$ & $-5.08(5.95)$ & 1.000 & 0.005 & 0.072 & 0.909 \\
\hline Yes & $22.98(7.44)$ & $23.68(5.75)$ & $18.92(5.98)$ & $0.71(9.23)$ & $-4.76(5.98)$ & 1.000 & 0.006 & 0.076 & \\
\hline $\mathrm{P}^{1}$ & 0.599 & 0.524 & 0.426 & & & & & & \\
\hline
\end{tabular}

${ }^{1}$-value for group effect; ${ }^{2} P$-value for time effect (BSL to end of therapy measurement); ${ }^{3}$-value for time effect (end of therapy to 6 months measurement); ${ }^{4} P$-value for time effect (BSL to 6 months measurement); ${ }^{5}$ Repeated measurements ANOVA. Effects reported include significant differences between the two groups in the degree of change in each particular variable during the study period; ' analysis was based on logarithmic transformations, BSL, baseline; EOT, end of therapy; 6Mo-EOT, 6 months after the end of therapy; IL-17A, interleukin 17A; BAFF, B-cell activating factor; MC, mixed cryoglobulinemia

and vitamin $\mathrm{D}$ were found between groups with or without cirrhosis $(\mathrm{P}=0.363, \mathrm{P}=0.567, \mathrm{P}=0.956$, respectively) and those with low or high HCV RNA levels $(\mathrm{P}=0.737, \mathrm{P}=0.3, \mathrm{P}=0.317$, respectively).

\section{Discussion}

Several studies have suggested that cytokines play an important role in the pathogenesis, progression and treatment outcome of $\mathrm{HCV}$-infected patients. The immune responses are highly complex and not well defined. Extrahepatic autoimmune involvement with abnormal immune responses mediated by both $\mathrm{T}$ and B lymphocytes is observed in $\mathrm{CHC}$, mainly in those with cryoglobulinemia [21,22]. Nevertheless, the role of different IL-17A-secreting T cells on CHC patients with MC is less clear.

Th17 cells are a subpopulation of CD $4+\mathrm{T}$ cells that have been implicated in the disease progression of many autoimmune and inflammatory disorders [23]. In the present study, we compared IL-17A and BAFF levels between CHC patients with and without asymptomatic MC, as well as between $\mathrm{CHC}$ patients and HC. Both cytokines were found to be significantly elevated in MC compared to the non-MC group. Other studies evaluating the immune phenotype and its relationship with $\mathrm{B}$ cells in these patients have reported elevated serum BAFF levels in patients with MC [24,25]. To our knowledge, there is no other study showing increased IL-17A levels in MC, suggesting that IL-17A might participate in HCV-related autoimmunity. There is one study in which increased expression of IL-17 was associated with $\mathrm{HCV}$ infection but not with asymptomatic MC [26].

We observed a significant increase in the IL-17A levels in $\mathrm{HCV}$-infected patients compared to $\mathrm{HC}$, confirming recent reports $[27,28]$. Interestingly, further analysis showed high levels of IL-17A in patients who responded to antiviral treatment versus non-responders [29]. Hence, upregulation of IL-17A might be considered as a predictor of the response to therapy with pegIFN/RBV. It is difficult to propose a cutoff point and larger studies are required to further assess these findings. When we further analyzed IL-17A kinetics during the treatment period, we noticed that antiviral therapy significantly downregulated IL-17A in the SVR group (Fig. 1). This finding persisted in responders beyond the cessation of antiviral treatment, suggesting that the suppressed cytokine release could be attributed to the viral clearance itself and not to the antiviral treatment. Likewise, Sousa et al suggested that pegIFN/RBV treatment resulted in a significant downmodulation of IL-17, mainly in responders [30].

On the other hand, IL-17A was upregulated in the non-SVR group. Our results suggest that a worsening proinflammatory 
condition (IL-17A) during antiviral treatment may have a deleterious effect and may lead to treatment failure. The study of Fathy et al described a normalizing immune response during treatment concerning IL-17A, in both responders and non-responders, but in a very small number of patients and over a short period of time [31], while Sousa et al described a non-significant downregulation in their non-responder group. Menezes et al reported that patients with SVR displayed lower IL-17 levels compared with non-responders [32].

Innate and adaptive immunity are associated with the prognosis of HCV infection and the response to antiviral therapy. Suppressed cellular immunity against HCV may contribute to the development of chronic infection $[5,33]$. Similarly to what Lake-Bakaar et al described [34], the current study demonstrated that BAFF kinetics during antiviral treatment differed between responders and non-responders. On the other hand, unlike the earlier study, we included asymptomatic patients with MC and extended the investigation of BAFF kinetics to 6 months after the end of treatment. Interferon therapy in responders induced an enhanced immune response, as indicated by increasing BAFF levels, resulting in viral clearance. Previous investigators revealed that antiviral therapy induced increased serum BAFF values, irrespectively of treatment response [8].

It is well established that BAFF levels seem to be higher in patients with MC, especially when vasculitis is present. In the current study, which included asymptomatic patients with or without MC, BAFF levels were higher in the MC group, but they were comparable between patients and HC. Other studies also showed that HCV patients without vasculitis had levels similar to those of healthy individuals $[35,36]$. Higher BAFF levels compared to $\mathrm{HC}$ were evident in patients with cryoglobulinemia [34].

Vitamin $\mathrm{D}$ is an immune modulator that reduces inflammation while enhancing protective immune responses. Severe vitamin D deficiency (25-OH-vitamin D serum level $<20 \mathrm{ng} / \mathrm{mL}$ ) is a common feature in chronic hepatitis C, even in the absence of advanced liver fibrosis [37]. A high rate of HCV patients in the current study had vitamin D deficiency ( $<20 \mathrm{ng} / \mathrm{mL})$ or insufficiency (20-29 ng/mL). Increased IL$17 \mathrm{~A}$ values were evident in patients with MC compared to those without, but no difference in vitamin D levels at BSL was noticed between these groups. However, using mixed linear models we found that changes in IL-17A were significantly associated with changes in vitamin $\mathrm{D}$ levels $(\beta=-0.04, \mathrm{SE}=0.02$, $\mathrm{P}=0.046)$, indicating an interaction between vitamin $\mathrm{D}$ and IL17 expression [38].

This study had some limitations. First, the small size of the sample and HC group and the short post-treatment period of follow up may have prevented some results from reaching statistical significance. On the other hand, this study may serve as preliminary data for further investigation into this issue. Second, in the DAA era, the effect of antiviral treatment with pegIFN/RBV may appear outdated. However, many changes in the variables measured at BSL and at the EOT persisted for long after treatment, suggesting a normalization of the immune response achieved by viral clearance.
In conclusion, different kinetics of IL-17A, BAFF and vitamin $\mathrm{D}$ were assessed between responders and nonresponders to antiviral treatment resulting in normalization of the immune response in the former group after treatment. An interplay between IL-17A and vitamin D concentrations was apparent during the antiviral treatment period, a finding that may explain the low levels of vitamin $\mathrm{D}$ in this group of patients. Finally, overexpression of both BAFF and IL-17A is associated with the presence of $\mathrm{MC}$ in $\mathrm{CHC}$ patients. MC poses therapeutic challenges even in the DAA era. In this respect, any additional information on this topic definitely enhances our ability to understand the complex mechanisms involved in cryoglobulinemia and its effect on $\mathrm{HCV}$ patients.

\section{Summary Box}

\section{What is already known:}

- Mixed cryoglobulinemia (MC) in chronic hepatitis $\mathrm{C}(\mathrm{CHC})$ is associated with abnormal immune responses mediated by $\mathrm{T}$ and $\mathrm{B}$ cells

- Interleukin (IL)-17 and B-cell activating factor (BAFF) levels are higher in the serum of $\mathrm{CHC}$ patients than in healthy controls

- Treatment with pegylated interferon-a (pegIFN) and ribavirin (RBV) downregulates IL-17A and upregulates BAFF

- An increase in IL-17 is correlated with a significant decrease in vitamin $\mathrm{D}$

\section{What the new findings are:}

- IL-17A was shown to be significantly higher in $\mathrm{CHC}$ patients with MC

- High levels of IL-17A or low levels of BAFF appear to be associated with a sustained virological response to IFN/RBV

- Downregulation of IL-17A and upregulation of BAFF was observed after antiviral treatment in responders

- There is an interaction between vitamin D and IL$17 \mathrm{~A}$ levels associated with antiviral treatment

\section{References}

1. Petruzziello A, Marigliano S, Loquercio G, Cozzolino A, Cacciapuoti C. Global epidemiology of hepatitis $C$ virus infection: An up-date of the distribution and circulation of hepatitis $\mathrm{C}$ virus genotypes. World J Gastroenterol 2016;22:7824-7840.

2. Cacoub P, Gragnani L, Comarmond C, Zignego AL. Extrahepatic manifestations of chronic hepatitis C virus infection. Dig Liver Dis 2014;46(Suppl 5):S165-S173.

3. Schamberg NJ, Lake-Bakaar GV. Hepatitis C virus-related mixed cryoglobulinemia: pathogenesis, clinical manifestations, and new 
therapies. Gastroenterol Hepatol (N Y) 2007;3:695-703.

4. Ferri C, Giuggioli D, Cazzato M, Sebastiani M, Mascia MT, Zignego AL. HCV-related cryoglobulinemic vasculitis: an update on its etiopathogenesis and therapeutic strategies. Clin Exp Rheumatol 2003;21:S78-S84.

5. Shoukry NH, Grakoui A, Houghton M, et al. Memory CD8+ T cells are required for protection from persistent hepatitis $\mathrm{C}$ virus infection. J Exp Med 2003;197:1645-1655.

6. Ulsenheimer A, Gerlach JT, Gruener NH, et al. Detection of functionally altered hepatitis $\mathrm{C}$ virus-specific CD4 T cells in acute and chronic hepatitis C. Hepatology 2003;37:1189-1198.

7. Zignego AL, Ferri C, Giannelli F, et al. Prevalence of bcl-2 rearrangement in patients with hepatitis $\mathrm{C}$ virus-related mixed cryoglobulinemia with or without B-cell lymphomas. Ann Intern Med 2002;137:571-580.

8. Fabris M, Quartuccio L, Sacco S, et al. B-Lymphocyte stimulator (BLyS) up-regulation in mixed cryoglobulinaemia syndrome and hepatitis-C virus infection. Rheumatology (Oxford) 2007;46:37-43.

9. Naoumov NV. Hepatitis C virus-specific CD4(+) T cells: do they help or damage? Gastroenterology 1999;117:1012-1014.

10. Saadoun D, Bieche I, Maisonobe T, et al. Involvement of chemokines and type 1 cytokines in the pathogenesis of hepatitis $\mathrm{C}$ virus-associated mixed cryoglobulinemia vasculitis neuropathy. Arthritis Rheum 2005;52:2917-2925.

11. Klemann C, Schröder A, Dreier A, et al. Interleukin 17, produced by $\gamma \delta \mathrm{T}$ cells, contributes to hepatic inflammation in a mouse model of biliary atresia and is increased in livers of patients. Gastroenterology 2016;150:229-241.

12. Ray S, De Salvo C, Pizarro TT. Central role of IL-17/Th17 immune responses and the gut microbiota in the pathogenesis of intestinal fibrosis. Curr Opin Gastroenterol 2014;30:531-538.

13. Qian XJ, Zhu YZ, Zhao P, Qi ZT. Entry inhibitors: New advances in HCV treatment. Emerg Microbes Infect 2016;5:e3.

14. European Association for the Study of the Liver. EASL Recommendations on Treatment of Hepatitis C 2018. J Hepatol 2018;69:461-511.

15. Roccatello D, Sciascia S, Rossi D, et al. The challenge of treating hepatitis $C$ virus-associated cryoglobulinemic vasculitis in the era of anti-CD20 monoclonal antibodies and direct antiviral agents. Oncotarget 2017;8:41764-41777.

16. Bian DD, Zhou HY, Liu S, et al. Current treatment status and barriers for patients with chronic HCV infection in mainland China: A national multicenter cross-sectional survey in 56 hospitals. Medicine (Baltimore) 2017;96:e7885.

17. Wei R, Christakos S. Mechanisms Underlying the Regulation of Innate and Adaptive Immunity by Vitamin D. Nutrients 2015;7:8251-8260.

18. Arteh J, Narra S, Nair S. Prevalence of vitamin D deficiency in chronic liver disease. Dig Dis Sci 2010;55:2624-2628.

19. Backstedt D, Pedersen M, Choi M, Seetharam A. 25-Vitamin D levels in chronic hepatitis $C$ infection: association with cirrhosis and sustained virologic response. Ann Gastroenterol 2017;30:344-348.

20. Zeuzem S, Rodríguez-Torres $M$, Rajender Reddy $K$, et al. Optimized threshold for serum HCV RNA to predict treatment outcomes in hepatitis C patients receiving peginterferon alfa-2a/ ribavirin. J Viral Hepat 2012;19:766-774.

21. Ramos-Casals M, Jara LJ, Medina F, et al; HISPAMEC Study Group. Systemic autoimmune diseases co-existing with chronic hepatitis $\mathrm{C}$ virus infection (the HISPAMEC Registry): patterns of clinical and immunological expression in 180 cases. J Intern Med 2005;257:549-557.
22. Fadda SH, Bassyouni IH, Hamdy A, Foad NA, Wali IE. Anti-C1q in chronic hepatitis $\mathrm{C}$ virus genotype IV infection: association with autoimmune rheumatologic manifestations. Immunol Invest 2015;44:45-55.

23. Sandquist I, Kolls J. Update on regulation and effector functions of Th17 cells. F1000Res 2018;7:205.

24. Sene D, Limal N, Ghillani-Dalbin P, Saadoun D, Piette JC, Cacoub P. Virus-associated B-cell proliferation - the role of serum B lymphocyte stimulator (BLyS/BAFF). Rheumatology (Oxford) 2007;46:65-69.

25. Toubi E, Gordon S, Kessel A, et al. Elevated serum B-lymphocyte activating factor (BAFF) in chronic hepatitis $\mathrm{C}$ virus infection: association with autoimmunity. J Autoimmun 2006;27:134-139.

26. Kong F, Zhang W, Feng B, et al. Abnormal CD4+T helper (Th) 1 cells and activated memory B cells are associated with type III asymptomatic mixed cryoglobulinemia in HCV infection. Virol J 2015; 12:100.

27. Kondo Y, Ninomiya M, Kimura O, et al. HCV infection enhances Th17 commitment, which could affect the pathogenesis of autoimmune diseases. PLoS One 2014;9:e98521.

28. Chang Q, Wang YK, Zhao Q, Wang CZ, Hu YZ, Wu BY. Th17 cells are increased with severity of liver inflammation in patients with chronic hepatitis C. J Gastroenterol Hepatol 2012;27:273-278.

29. Meng P, Zhao S, Niu X, et al. Involvement of the interleukin-23/ interleukin-17 axis in chronic hepatitis $\mathrm{C}$ virus infection and its treatment responses. Int J Mol Sci 2016;17:1070.

30. Jimenez-Sousa MA, Almansa R, de la Fuente C, et al. Increased Th1, Th17 and pro-fibrotic responses in hepatitis C-infected patients are down-regulated after 12 weeks of treatment with pegylated interferon plus ribavirin. Eur Cytokine Netw 2010;21:84-91.

31. Fathy A, Ahmed AS, Metwally L, Hassan A. T helper type 1/T helper type 17-related cytokines in chronic hepatitis $C$ patients before and after interferon and ribavirin therapy. Med Princ Pract 2011;20:345-349.

32. Menezes EG, Coelho-dos-Reis JGA, Cardoso LM, et al. Strategies for serum chemokine/cytokine assessment as biomarkers of therapeutic response in $\mathrm{HCV}$ patients as a prototype to monitor immunotherapy of infectious diseases. Antiviral Res 2017;141:19-28.

33. Grakoui A, Shoukry NH, Woollard DJ, et al. HCV persistence and immune evasion in the absence of memory $\mathrm{T}$ cell help. Science 2003;302:659-662.

34. Lake-Bakaar G, Jacobson I, Talal A. B cell activating factor (BAFF) in the natural history of chronic hepatitis $\mathrm{C}$ virus liver disease and mixed cryoglobulinaemia. Clin Exp Immunol 2012;170:231-237.

35. Atta AM, Oliveira IS, Sousa GM, Paraná R, Atta ML. Serum cytokine profile in hepatitis $\mathrm{C}$ virus carriers presenting cryoglobulinaemia and non-organ-specific autoantibodies. Microb Pathog 2010;48:53-56.

36. Landau DA, Rosenzwajg M, Saadoun D, Klatzmann D, Cacoub P. The B lymphocyte stimulator receptor-ligand system in hepatitis C virus-induced B cell clonal disorders. Ann Rheum Dis 2009;68:337-344.

37. Lange CM, Bojunga J, Ramos-Lopez E, et al. Vitamin D deficiency and a CYP27B1-1260 promoter polymorphism are associated with chronic hepatitis $\mathrm{C}$ and poor response to interferon-alfa based therapy. J Hepatol 2011;54:887-893.

38. Schaalan MF, Mohamed WA, Amin HH. Vitamin D deficiency: correlation to interleukin-17, interleukin-23 and PIIINP in hepatitis C virus genotype 4. World J Gastroenterol 2012;18:3738-3744. 